\title{
Administración y calidad de la información de los sistemas de información contable de las PYMES
}

\author{
Management and information quality of SME's accounting information systems \\ Gestion et qualité de l’information des systèmes d’information comptable des PME
}

\begin{abstract}
José M. Medina-Quintero
Profesor-Investigador de la Universidad Autónoma de Tamaulipas,

Victoria Tamaulipas - México.

PhD en Sistemas de Información de la Empresa.

E-mail: jmedinaq@uat.edu.mx

Perla E. Aguilar-Gámez

Profesora, Maestra en Dirección Empresarial, Facultad de Comercio y Administración-Victoria Universidad Autónoma de

Tamaulipas, Victoria Tamaulipas - México.

E-mail: edith_gamesin@hotmail.com

Artículo de investigación científica y tecnológica según clasificación COLCIENCIAS

Recepción: 18/02/2013

Corrección: 13/03/2013

Aprobación: 04/04/2013
\end{abstract}

\section{Resumen}

La información se manifiesta como un factor de incidencia para las organizaciones en la concepción y persistencia de ventajas competitivas. Sin embargo, en últimas fechas se ha vuelto indispensable que la información esté bien administrada y sea de buena calidad. Esta investigación analiza el grado de influencia que tiene la administración y la calidad de la información en los sistemas de información contable (SIC) de las pequeñas y medianas empresas (PYMES) para la toma de decisiones y su uso/utilidad por los usuarios. El estudio empírico se lleva a cabo en la región centro del estado de Tamaulipas (México) por medio del análisis de regresión con el software PASW Statistics. Los resultados muestran el impacto que tiene la administración de la información para el uso/utilidad de la información contable. Asimismo, la calidad de la información influye en la toma de decisiones y en el uso/utilidad de la información contable. Cabe resaltar que la administración de la información no incide para la toma de más y mejores decisiones, se requiere profundizar en la relación al ser estos hallazgos el resultado de una investigación en un grupo de PYMES, por lo que no se pueden generalizar a todas las organizaciones de este tipo.

Palabras clave: sistemas de información contable, administración de la información, calidad de la información, toma de decisiones.

\begin{abstract}
Information is manifested as an influential factor in the conception and maintenance of organizations' competitive advantage. However, the degree of influence that information has upon other functions in organizations depends on its management and quality. This research analyzes the degree of influence information management and quality has on decision making and the use/usefulness of accounting information systems' (AIS) in small and medium sized enterprises (SMEs) for users. The empirical study took place in the central region of the state of Tamaulipas, Mexico by means of a regression analysis with the aid of the PASW Statistics software. Results show the impact information management and quality have on the use/usefulness of the accounting information. Similarly, they also show the impact information quality has on the users' decision-making processes and use/usefulness for the accounting information. A surprising finding is that good information management does not necessarily contribute to making more and better decisions. A more in-depth study of the relationships between these two constructs is required, given that these findings are the result of research in a group of SMEs. Therefore, they are not generalizable to other organizations of the same type.
\end{abstract}

Keywords: accounting information systems, information management, information quality, decision making.

\section{Résumée}

L’information se manifeste comme un facteur d’incidence pour les organisations sur la conception et la persistance d’avantages compétitives. Pourtant, actuellement il est devenu indispensable que l`information soit bien gérée et de bonne qualité. Cette recherche analyse le degré d’influence de la gestion et la qualité de l’information sur les systèmes d`information comptable (SIC) dans les petites et moyennes entreprises (PME) pour la prise de décisions et leur usage/utilité pour les utilisateurs. Ľétude empirique se développe dans la région centrale de l'état de Tamaulipas (Méxique) à partir de l'analyse de régression avec le software PASW Statistics. Les résultats montrent l'impact de la gestion de l’information sur l’usage/utilité de l’information comptable. De même, c’est possible d’observer que la qualité de l’information 
influence la prise de décisions et l'usage/utilité de l'information comptable. Il faut bien remarquer que la gestion de l'information n`a pas d’incidence sur la prise de plus et de meilleures décisions, mais il est nécessaire d'approfondir sur cette relation puisque ces découvertes sont le résultat d`une recherche sur un groupe de PME, ce qui ne permet pas de généraliser sur toutes les organisations de ce type.

\section{Mots clef: systèmes d'information comptable, gestion de} l'information, qualité de l'information, prise de décisions.

\section{Introducción}

En la actualidad, las instituciones emplean la información y los sistemas de información (SI) como herramientas para llevar la delantera a sus competidores; no obstante, el éxito de la empresa no depende solo de cómo maneje sus recursos materiales.

La idea de la información es un recurso a desarrollar, utilizar, reutilizar como negociable, así se muestra en una serie de publicaciones popularizadas por Alvin Toffler en La tercera ola, donde postula un tercer período de industrialización, basado en el campo de la información, sin embargo, si la información realmente se puede considerar como un recurso, también es una cuestión espinosa.

Por otra parte, mientras que las pequeñas empresas han sido tradicionalmente vistas como renuentes a invertir en SI, las evidencias muestran un aumento en la última década en la conciencia de los usuarios y la administración de sistemas informáticos en las pequeñas empresas por los propietarios y gerentes.

En este sentido, la administración de la información puede ser considerada como el control de un proceso o procesos hacia un estado objetivo o meta específica. De acuerdo con lo dicho por Best (2010), quien señaló que hay una conciencia creciente de que la información puede ser considerada como un recurso a ser administrado colectivamente, e independientemente de su tecnología, la información puede proporcionar ventajas competitivas importantes y pueden mejorar el rendimiento general de la organización.

El camino a seguir para los administradores es por lo tanto, desarrollar técnicas y herramientas que permitan a la dirección general guiar en el uso y desarrollo de los datos, considerando que los usuarios toman sus decisiones con base en la información de calidad obtenida de los SI.

Así, el correcto desarrollo de la organización depende de que exista un adecuado flujo de información entre ésta y su contexto, y entre las distintas unidades que la conforman, pensando que una empresa es más competitiva cuanto se destaca en la explotación de la información del entorno.

La información se ha distinguido por ser una parte importante en toda organización, infortunadamente las empresas no han aprovechado todos esos datos generados diariamente en las instituciones para tomar mejores decisiones, o simplemente, no saben si esa información les está siendo útil no solo para obtener ventaja competitiva sino también el conocer si realmente todos los procesos que se llevan a cabo con los SI son útiles para el desarrollo organizacional.

Con lo anterior, surgen las preguntas de investigación: ¿Cómo deben las PYMES gestionar sus recursos de información que les permita tomar más y mejores decisiones? ¿Cómo se debe presentar la información de los sistemas de información contable (SIC) a sus usaurios para que les sea de utilidad y no simplemente responder a una necesidad inmediata del mercado? ¿Cómo se puede justificar el control de calidad de la información generada en un SIC para mejorar la toma de decisiones y la utilidad?

Partiendo de lo antes expuesto, el objetivo de la presente investigación es determinar el grado de influencia que tienen la administración y la calidad de la información de los SIC que usan las pequeñas y medianas empresas, para tomar más y mejores decisiones y hacer un mejor uso/utilidad de estos sistemas por parte de sus usuarios. Ello hace delimitar al hecho de conocer alguna relación entre las variables independientes y dependientes, las cuales se operacionalizan en el apartado de Metodología. Esta investigación ronda en torno a esta operacionalización y no considera constructos importantes como la satisfacción de usuarios, participación de directivos y usuarios, calidad del sistema y de los servicios, incluso no pretende sugerir la forma de cómo se deben tomar las decisiones ni mucho menos, cómo puede ser más útil el SIC, porque solo presenta la imagen de un momento en el uso y aplicación de estos SI por parte de sus usuarios.

\section{Revisión de literatura}

\subsection{Toma de decisiones}

Este concepto se define como la selección de un curso de acción entre alternativas. La decisión tiene que estar enlazada con otras actividades (Koontz y Weihrich, 2004), en otras palabras, la generación, evaluación y selección de soluciones (Huber y McDaniel, 1989) en forma racional. Los SI abarcan todo el rango de operaciones y las actividades de toma de decisiones (Miller y Doyle, 1987), en sí, es su filosofía, complicándose de esta manera. Otros investigadores como Teng y Calhoun (1996) señalan que el efecto potencial de la tecnología de información (TI) en la toma de decisiones en todos los niveles ha sido capturado por los practicantes de los SI desde principios de la era informática, porque el mundo se mueve hacia mercados abiertos y globales, la necesidad por el acceso a información oportuna, confiable y fácil será la clave para la efectiva toma de decisiones (Leidner et al., 1999), de tal modo que los directivos deben determinar si los SI ayudan a lograr las metas de toma de decisiones.

La toma de decisiones es uno de los roles más importantes de los ejecutivos, también usuarios, en especial 
cuando este proceso en una organización es multidimensional (Teng y Calhoun, 1996), porque la importancia de una decisión es el impacto de ésta en la empresa y en el desempeño a largo plazo (Tzu-Chuan et al., 1998). La calidad de la información permite a un tomador de decisiones justificar las bases de las decisiones, argumentando que si la información usada es oportuna, exacta y confiable, entonces, cualquier decisión hecha es buena (O'Reilly, 1982). Para sistemas que ayudan a tomar decisiones o proveer un servicio al usuario, es mucho más difícil estimar los beneficios, habiendo pocos ejemplos de estos intentos, eso sí, la calidad de la decisión se ha encontrado que está más cercanamente relacionada con la participación actual del usuario.

En muchas organizaciones, las responsabilidades de toma de decisiones se han descentralizado para permitir mayor control, autoridad y autonomía a los trabajadores (Sherman et al., 2004) tomando en cuenta que un esfuerzo es exitoso si dirige a (Anderson y Narasimhan, 1979):

* Una transformación de patrones de respuesta de decisiones (por ejemplo, decisiones diferentes, procedimientos diferentes para la toma de decisiones, uso diferente de la información o uso de información no existente previamente).

* Un gran entendimiento de la situación de la decisión y los conceptos usados.

* Beneficios operativos como alta rentabilidad.

* Incremento en la confianza y menos tiempo para tomar alguna decisión en particular.

Si se considera que los usuarios toman sus decisiones con base en la información de calidad obtenida de los SI y aunado a la urgencia de generar toma de decisiones rápidas en todos los sectores productivos (Medina y Chaparro, 2007), muchas organizaciones no han modernizado sus sistemas informáticos tanto en hardware como en software y continúan con software obsoleto creado en la década de 1980 o sistemas que no se adecúan a sus verdaderas necesidades.

\subsection{Uso/utilidad}

Un SIC comprende una colección de datos y procedimientos de procesamiento contable que genera la información necesaria para sus usuarios a fin de crear cursos de acción, siempre pensando en el beneficio de la organización. El uso del sistema de información contable y la percepción de efectividad permiten definir un rol clave en la determinación de su efectividad (Srinivasan, 1985), pero si solo se dice que el uso traerá más beneficio sin razonar su naturaleza, es claramente insuficiente (DeLone y McLean, 2002). Por una parte, la variable uso se refiere a la inclusión de la información generada por el sistema en el proceso de toma de decisiones por el usuario (Cheney y Dickson, 1992). Por otro lado, en el caso de efectividad, se mide por el uso de la información proveída por el SIC expresada en términos de satisfacción del usuario y la calidad de las decisiones hechas (Serafeimidis, 2002).

El uso del SIC y su efectividad pueden indicar fenómenos completamente distintos (Srinivasan, 1985); pero el uso, es una variable central en la investigación del SI (DeLone y McLean, 2003) que se ha definido y conceptualizado como el número de veces que se utiliza un SI porque una empresa que paga por un sistema que usa y no apoya a los objetivos del negocio, no tiene éxito, es como si no lo usara (Gelderman, 1998) y ha hecho una mala inversión (Markus y Keil, 1994). Estos últimos investigadores arguyen también que su uso es inevitable cuando el interés de desarrolladores y de los usuarios está alineado con buenos diseños de los sistemas, aunque Tzu-Chuan et al. (1998) manifiesta que en ocasiones se complica cuando los aspectos políticos y sociales se involucran.

La operación del SIC se cree que es una conducta que refleja una expectación de los beneficios netos por ese uso, proporcionando un resultado conductual del éxito del sistema (Rai et al., 2002), pero uno de los problemas más serios es la presunción si la forma en que se prueba el sistema será igual en el uso y operación real (Jeske y Zhang, 2005). Si el usuario considera que el SIC no es confiable y sus datos inexactos, esas dudas se reflejarán en su uso (o no uso) (Ives et al., 1983), pero cuando la satisfacción impacta el uso, con un alto nivel, construye una mayor dependencia en el sistema. Se agrega a lo anterior, que las personas con más experiencia tienen un promedio más alto de usar un SI (Mathieson y Chin, 2001), también el uso pasado de un sistema aparentemente influye en su facilidad de operación y es el factor clave en la determinación de uso futuro (Davis, 1989), más precisamente en su satisfacción general (Molla y Licker, 2001). Por tanto, esta variable ha sido con frecuencia utilizada por los investigadores del SI como una medida del éxito del sistema para determinar los beneficios de la tecnología (Igbaria et al., 1995), pero cuando se usa de manera aislada, no puede medir completamente el éxito (DeLone y McLean, 2003).

El uso puede preceder a la satisfacción del usuario en un sentido de proceso pero la experiencia positiva con el uso dirige a la satisfacción en un sentido causal. Similarmente, la satisfacción del usuario puede incrementar la intención de uso y el uso mismo (DeLone y McLean, 2002). Según Straub, Limayen y Karahanna-Evaristo (1995) la forma más común de medir el uso en la literatura es:

* Subjetiva, medición de autoreporte, y

* Objetiva, medición de registro de computadora.

Aunado a esto, los estudios publicados en las ciencias sociales indican que las medidas subjetivas tienen indicadores no del todo concluyentes ni aceptables. Y para diagnosticar el uso de un SI en un contexto organizacional, Auer y Rouhonen (1997), proponen analizar: 
* Uso: frecuencia de uso, tipos.

* Habilidades: operacionales, de desarrollo y mantenimiento, de soporte.

* Conocimiento: conceptos de hardware, de software, políticas de los SI organizacionales, SI potenciales y aplicaciones existentes.

* Vistas: voluntad para usarlos, habilidades y conocimientos de desarrollo, responsabilidades de los SI.

* Habilidades y conocimiento organizacional: conocimiento interpersonal, de conducta, dinámica de grupos, objetivos, administración de objetivos, etc.

Por otra parte, muchas causas potenciales del no uso de los sistemas son previsibles, atribuible a dos factores: i) implementación (el staff debe asegurarse que el sistema se use) y ii) utilidad del software (amigable con el usuario) (Markus y Keil, 1994). Al mismo tiempo, muchos sistemas no son diseñados para la implementación a fin de mejorar el desempeño individual y organizacional; en esta idea es importante encontrar el porqué los individuos eligen o no usar un SI (Mathieson y Chin, 2001). Así, por ejemplo, en los resultados de Srinivasan (1985), muestran que quienes pasan por largos períodos frente a la computadora, tienden a ver el sistema como que no contribuye favorablemente a sus operaciones; al igual que Gelderman (1998) quien no encontró una relación significativa entre el uso del sistema y el desempeño del usuario.

Por último, el Modelo de Aceptación Tecnológica (TAM, por sus siglas en inglés de Technology Acceptance Model) introducido por Davis (1986) intenta explicar la conducta del uso de la computadora, describiendo la relación entre utilidad percibida y la percepción de facilidad de uso, y la actitud del usuario, intenciones y la conducta de uso (Straub et al., 1995), porque la gente cree que la tecnología le ayudará a producir su trabajo mejor (Davis, 1986) y si la utilidad percibida y el nivel de servicios es bueno, entonces el uso del sistema es más intensivo (Sääksjärvi y Talvinen, 1993).

\subsection{Administración de la información}

Robbins y Coulter (2010) definen a la administración como la coordinación y supervisión de las actividades laborales de otras personas de tal manera que se realice de forma eficiente y eficaz. Por su parte, los SI responden a tareas básicas necesarias para llevar a cabo la actividad fundamental de cualquier empresa. Estas tareas son necesarias simplemente para coordinar las acciones de carácter operativo como lo es la administración de la información (Al) que se define como la producción, el control, el almacenamiento, la recuperación y distribución de información externa e interna de manera económica, eficiente y eficaz, a fin de mejorar el desempeño de una organización (Best, 2010).
Es preciso señalar que en la actualidad, la mayoría de las empresas depende de las TI para el manejo exacto y oportuno de la información. La contabilidad genera muchos datos: registros contables, estados financieros, entradas, salidas, etc., pero surge la pregunta, ¿qué hago con ellos? En el sentido de contribuir a la efectividad organizacional, su impacto (de datos e información contable) está constantemente oculto hasta que se remueve o se pierde (Oppenheim et al., 2004), es decir, la información tiene sentido solo cuando alguien la usa para algo. En esta idea, el uso de la información contable por medio de la tecnología se ha demostrado que tiene una influencia positiva en el desempeño (Shin, 2001), porque cuando los clientes o usuarios encuentran un producto de calidad útil, les permite ejecutar exitosamente la acción para la cual la información fue desarrollada.

La aparición de la información como factor productivo y motor de desarrollo ya se está haciendo evidente en la sociedad, donde cada vez se dedica más tiempo y recursos de forma directa e indirecta a su tratamiento, para el proceso y entrega de reportes, adquisición de datos, y transferencia y carga, lo que trae como consecuencia que el valor de la TI dependa enormemente de los tipos de datos usados.

En suma, la Al es el punto central donde convergen datos e información en muchas empresas, las cuales han incrementado el uso de la TI para almacenar, recuperar documentos y elementos de conocimiento codificado para apoyar la gestión y compartición del conocimiento tácito y explícito (OECD, 2004). Por lo tanto, la información juega un papel crucial en la facilitación del proceso de intercambio con la cadena de valor como parte de la estrategia del negocio (Davenport, 1997) porque los usuarios la necesitan para entender el significado de los datos (English, 2001) y virtualmente, todos en la empresa (operadores, ejecutivos, etc.) usan información para producir otra nueva.

Indudablemente, la información es un componente intrínseco en casi todas las actividades de la organización al punto de tornarse transparente, porque es la vía por medio de la cual las personas expresan, representan, comunican y comparten sus conocimientos. Marchand et al. (2002) destacan que es el uso de la información lo que incide en la creación de valor del negocio por medio de cuatro prioridades estratégicas: i) minimizando riesgos financieros, comerciales y operacionales, ii) reduciendo costos de transacciones y procesos, iii) agregando valor a clientes y mercados, y iv) creando nuevas realidades por medio de la innovación.

Diversos estudios (DeLone y McLean, 2002, 2003; Shin, 2003; Medina y Chaparro, 2007) han dicho que la utilidad de la información es un factor adquirido, representa una variable para la dimensión de la calidad de la información, y de acuerdo con Franz y Robey (1986) este concepto tiene la capacidad de apoyar a los usuarios finales a satisfacer sus requerimientos y necesidades, 
también está asociada con la percepción de utilidad de la información. Davis (1989) agrega que se relaciona con el grado en el cual el uso de un sistema en particular puede ayudar a ejecutar mejor el trabajo de un usuario, induciendo que una buena información dirige a una buena toma de decisiones (O’Reilly, 1982).

Por lo anterior, el gerente de información de la empresa, debe tomar en cuenta las funciones de manejo o ser responsable del procesamiento de la información en la organización. Por un lado, debe comprobar que la estructura organizativa coincida con la misión y visión empresarial, y por el otro, que las estrategias organizacionales sean compatibles con las estrategias de tecnología y procesamiento de la información.

Las pautas que se pueden alcanzar en cuanto a la dirección a seguir para obtener información, es que los sistemas de administración de la información deben controlar, valorar, medir el flujo de información y la auditoría de los sistemas que la procesan. El camino a tomar para la administración de la información es por tanto, desarrollar técnicas y herramientas en las áreas de la administración y la contabilidad que le permitirán a la dirección general guiar en el uso y desarrollo de la información de la misma manera que guía el uso y desarrollo de los recursos de otros en la empresa. Por consiguiente, las dos primeras hipótesis que se plantean en esta investigación son:

$\mathrm{H}_{1}$. La administración de la información de los SIC permite que los usuarios tomen más y mejores decisiones.

$\mathrm{H}_{2}$. La administración de la información de los SIC genera que los usuarios hagan un mayor uso y les sea más útil la información contable.

\subsection{Calidad de la información}

En la década de 1990, la liberalización de los mercados, las nuevas tecnologías, el incremento de la competencia y la necesidad de realizar drásticas reducciones de costos, hicieron surgir en muchas empresas programas de implantación de sistemas de gestión de calidad total, con el objetivo fundamental de aumentar la competitividad y de satisfacer las expectativas de los clientes.

No obstante, el aseguramiento de la calidad sigue considerándose una cuestión que se vincula, en esencia, al departamento de producción. Esta visión cambia al determinar la calidad como un factor estratégico. Ya no se trata de una actividad inspectora sino preventiva: planificar, diseñar, fijar objetivos, educar e implementar un proceso de mejora continua. La gestión estratégica de la calidad hace de ésta una fuente de ventajas competitivas que requiere del esfuerzo colectivo de todas las áreas y miembros de la organización.

Los recientes actos de corrupción en algunas empresas del mundo, han hecho crítica la evaluación de los datos de contabilidad en las organizaciones, sobre todo en la información financiera (Krishnan et al., 2005), además, estos últimos investigadores creen también que la confiabilidad de los datos de un SIC se enfoca en la evaluación de la estructura de control interno más que en su diseño. Al respecto, los principios de calidad de Deming, Ishikawa, Juran, Crosby y otros, se usan en la mejora de la calidad de los productos, pero en la actualidad también se han aplicado en la administración de la información (English, 2001), en concreto a los problemas de producción de informes de calidad, porque cada producto de información tiene un valor intrínseco para el usuario.

De ahí que la calidad de la información (Cl) se define como la medición de las salidas de datos de las TI en términos de ser exacta, oportuna, completa, confiable, relevante y precisa (DeLone y McLean, 2003), pero según Lillrank (2003) la definición más ampliamente usada es dada por la Sociedad Americana para la Calidad (ASQ, por sus siglas en inglés de American Society for Quality) y la de la ISO 9000-2000, que están basadas en la satisfacción del cliente, donde se puede lograr no sólo por cumplir con los requerimientos sino también por características inherentes del producto o servicio y las formas de su presentación a los usuarios. Sin embargo, como en los sistemas de manufactura, la calidad de la información basada en computadora se está haciendo trascendental para muchas organizaciones (Ballou, Wang, Pazer y Tayi, 1998) proveyendo grandes cantidades de ésta, pero pocos conocen las herramientas para manejarla eficientemente.

Los datos e información producidos por los SIC usados para planear, analizar, administrar, dirigir y controlar las operaciones del negocio se volvieron importantes desde hace mucho tiempo. De acuerdo con Krishnan et al. (2005), la falta de definiciones conceptuales formales y reglas de decisión hacen difícil desarrollar sistemas prácticos de evaluación de la confiabilidad de los datos. Sin duda, el concepto de Cl es un tema de actualidad que ninguna organización puede descuidar o ignorar, porque en los tiempos modernos su dependencia es vital, definida como apta para usarse, sin una aceptación universal (Juran y Godfrey, 1999) y difícil de medirla usando este término tan amplio.

Por lo tanto, la $\mathrm{Cl}$ es crítica para las organizaciones, y a pesar de décadas de investigación y práctica, el campo adolece de métodos compresivos para su evaluación y mejoramiento, sin una propuesta sistemática (Ballou et al., 1998), siendo esencial la necesidad de una metodología que evalúe cómo las organizaciones desarrollan sus productos de información, porque los directivos se siguen quejando de que las TI no han aumentado la calidad de su suministro de información, ya sea interna o externa. Esta situación de igual manera la señala Goff (2003), quien encontró que los expertos estiman que del $10 \%$ al $30 \%$ de la información de las empresas que fluye a través de los sistemas es deficiente (inadecuada, inconsistente, mal capturada, etc.).

Los usuarios viven en un ambiente rico en información, mucho más que antes y para las organizaciones del sector público o privado quienes viven en un ambiente competitivo, la calidad de la información es una manera de sobrevivir y generar ventaja competitiva, tomando en cuento lo dicho por Teo y Wong (1998) quienes encontraron que está positivamente relacionada con el trabajo, la satisfacción directiva y 
el impacto organizacional, de donde se desprenden las dos últimas hipótesis de esta investigación:

$\mathrm{H}_{3}$. La calidad de la información promueve un impacto positivo en los usuarios de los SIC para la toma de decisiones.

$\mathrm{H}_{4}$. La calidad de la información produce que los usuarios tengan un impacto positivo en el uso y la utilidad de los SIC.

\section{Metodología}

La tecnología está creciendo a un ritmo acelerado y la industrialización del siglo XX hizo que las empresas se hicieran grandes y complejas y cuando las instituciones crecen, requieren ajustarse a las nuevas revoluciones mundiales, en estos tiempos, a la revolución informática, por tanto, es primordial que junto con los individuos se integren y adapten las nuevas TI. Los SIC no quedan fuera de este ambiente, se requiere de ellos la información que generan para que sea aplicada en beneficio del desempeño de la organización, sin embargo, no se puede confiar ciegamente en sus resultados si se duda que estén proporcionando la información correcta y de calidad. Además, se requiere aprender a hacer un uso efectivo de esa información, a planificarla, a integrarla, en sí, a administrarla.

El objetivo de esta investigación es determinar la influencia que tiene la Al y la $\mathrm{Cl}$ en los SIC de las PYMES para la toma de decisiones y su uso/utilidad por los usuarios. El proceso seguido para alcanzarlo, inició con la revisión del estado de arte en cuanto a las variables independientes (administración de la información y calidad de la información) y las dependientes (toma de decisiones y uso/ utilidad). Se construyó el modelo de investigación (Figura 1), basado en la revisión de la literatura. Las variables se operacionalizan de la siguiente forma:

*Variables independientes: Administración de la información (importancia debida de la aplicación, aplicación estratégica de la información, mejoramiento de procesos), calidad de la información (exacta, oportuna, completa, consistente).

* Variables dependientes: Toma de decisiones (relevante, de calidad, evaluación de alternativas, rapidez) y uso/ utilidad (reportes conveniente para uso óptimo, mejoramiento de desempeño, incremento de la efectividad, información útil).

Antes de continuar, hay que dejar de manifiesto que las variables analizadas en la revisión de literatura, no permiten definir claramente las relaciones propuestas en esta investigación, porque éstas no provienen de una teoría sencilla de administración y calidad de la información con constructos de desempeño, en este caso la toma de decisiones y el uso/ utilidad.
Para la recolección de los datos, se diseñó un cuestionario, que fue revisado por profesionales en el área. Después de ser validado por académicos y expertos, el siguiente paso consistió en la realización de un estudio piloto, lo que ayudó a establecer la validez de los ítems y el contenido, en otras palabras, la aplicación del pretest del instrumento para mejorarlo, solicitando retroalimentación de los posibles errores. La principal aportación fue la eliminación de ítems que no tenían la suficiente confiabilidad. El resultado fue la determinación de cuatro ítems para las variables calidad de la información, toma de decisiones y uso/utilidad, además de tres para la variable administración de la información. El total de ítems final fue de 78 , todos ellos valorados en una escala de Likert de 5 puntos (Muy en desacuerdo... Muy de acuerdo). Es preciso hacer la aclaración que esta investigación es parte de un proyecto institucional que está en proceso de desarrollo, por ello el número total de ítems.

Con el cuestionario validado, se aplicó a profesionales que usan un SIC, ya sea un paquete comercial, hecho a la medida por la propia empresa o bien desarrollado por otra organización. Las mediciones perceptuales fueron iguales para todos los ítems. Para mayo de 2012, según el Sistema de Información Empresarial Mexicano (http://www.siem.gob. $\mathrm{mx}$ ), en el estado de Tamaulipas se contaba con 1,418 PYMES en la zona de estudio (Cd. Victoria) 152. La versión definitiva fue aplicada a 92 cuestionarios en 46 empresas que cuentan con un SIC. Los datos de las empresas participantes en el estudio, se conservan en anonimato porque así se convino con los gerentes o dueños de las mismas.

Con base en la información obtenida, se deriva la descripción general y analítica inferencial por medio del análisis de regresión con PASW Statistics versión 18, a fin de comprobar las hipótesis diseñadas. Por último, se derivó el desarrollo de las conclusiones tomando como base los análisis anteriores. Asimismo, los valores mínimos aceptados para la confiabilidad de ítems será el Alfa de Cronbach igual o superior a 0.7 (Nunnally, 1978), $R^{2}$ indica la varianza explicada por la variable dentro del modelo, la cual de acuerdo con Chin (1998) debería ser >0.1, porque valores menores, aún siendo significativos, proporcionan poca información y $\mathrm{R}$ representa la relación entre las variables, y para ser consideradas significativas, deberían alcanzar al menos un valor de 0.2 e idealmente situarse por encima de 0.3. La significancia (Sig.) debe ser $<0.05$.

\section{Resultados y discusión}

En primera instancia se realizó el análisis descriptivo. En cuanto al sexo, se obtuvo que en su mayoría son mujeres $(67 \%)$ quienes operan los SIC, que a la larga puede ser un elemento que los programadores de sistemas deben considerar, sobre todo, que participen activamente en el diseño y desarrollo del software, ya que pueden proporcionar ideas o aspectos que no han sido pensados con anterioridad.

En cuanto al grado de estudios se refiere, $87 \%$ se encuentra dentro del nivel licenciatura (pregrado). Lo que indica que 
las personas que están utilizando los SIC cuentan con los conocimientos necesarios para llevar a cabo el trabajo de operación de esta TI.

El tiempo que las personas tienen trabajando para la empresa en el manejo de los SIC es relativamente corto, de 1 a 5 años (59\%), para ello, se observa que en los departamentos o áreas de contabilidad hay una alta rotación de personal. Y en cuanto a la cantidad de horas aproximadas a la semana que el usuario usa el SIC, se manifiesta un resultado mayor para el lapso de 11 a 20 horas ( $37 \%)$, es decir, pasan el suficiente tiempo en la operación de esta tecnología.

Para el análisis inferencial, en la Tabla 1, aparecen los resultados de confiabilidad, donde se puede notar que todas las variables sobrepasan los mínimos recomendados de 0.7 y el cuestionario en general alcanza un valor de 0.954 .

\begin{tabular}{|c|r|c|r|}
\hline Tabla 1. Análisis de confiabilidad & \multicolumn{1}{|c|}{$\begin{array}{c}\text { Alfa de } \\
\text { Variable }\end{array}$} & Variable & $\begin{array}{c}\text { Alfa de } \\
\text { Cronbach }\end{array}$ \\
\hline $\begin{array}{c}\text { Administración de la } \\
\text { información }\end{array}$ & 0.717 & Toma de decisiones & 0.929 \\
\hline Calidad de la información & 0.938 & Uso/utilidad & 0.910 \\
\hline \multicolumn{2}{|c|}{ Fuente: Medina-Quintero, J.M y Aguilar-Gámez, P.E. (2013). } \\
\hline
\end{tabular}

En la Tabla 2, se muestra la relación obtenida en cada una de las hipótesis, la varianza explicada $\left(R^{2}\right)$ y el nivel de significancia o de confianza. La $\mathrm{R}^{2}$ indica la validez convergente, es decir, si los ítems miden realmente lo mismo al explicar el monto de varianza que una variable captura de sus indicadores, lo que viene a corroborase con la $t$ Student o el nivel de significancia (Sig).

\begin{tabular}{|l|c|c|c|c|}
\hline \multicolumn{1}{|c|}{ Habla Hipótesis } & R & $\mathbf{R}^{\mathbf{2}}$ & Sig. & Comentarios \\
\hline $\mathrm{H}_{1}=$ A.I. $\rightarrow$ Toma de decisiones & 0.415 & 0.178 & 0.081 & Rechazada \\
\hline $\mathrm{H}_{2}=$ A.I. $\rightarrow$ Uso/utilidad & 0.563 & 0.318 & 0.001 & Aceptada \\
\hline $\mathrm{H}_{3}=$ C.I. $\rightarrow$ Toma de decisiones & 0.818 & 0.673 & 0.000 & Aceptada \\
\hline $\mathrm{H}_{4}=$ C.I. $\rightarrow$ Uso/utilidad & 0.760 & 0.580 & 0.000 & Aceptada \\
\hline Fuente: Medina-Quintero, J.M y Aguilar-Gámez, P.E. (2013).
\end{tabular}

A continuación, en la Figura 1 se detalla en forma gráfica el modelo de investigación evaluado. Se determina el nivel de significancia $\left({ }^{*}=.05=95 \%,{ }^{* *}=.01=99 \%\right.$ y $* \star *=.001=999 \%)$.

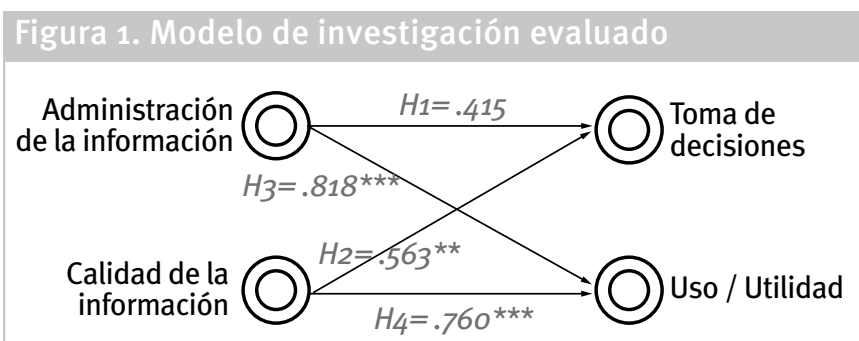

Fuente: Medina-Quintero, J.M y Aguilar-Gámez, P.E. (2013).

\section{Evaluación de hipótesis}

Como se observa, las hipótesis aceptadas al cumplir con los tres criterios ( $R, R^{2}$ y Sig.) son:

$H_{2}$ con un valor de $R=.563, R^{2}=.318$ y significancia $=.001$; por lo que se afirma que la administración de la información juega un papel importante, genera que los usuarios realicen un mayor uso de reportes que proveen información útil, además de mejorar el desempeño e incrementa la efectividad en el trabajo.

$\mathrm{H}_{3}$ con un valor de $\mathrm{R}=.818, \mathrm{R}^{2}=.673$ y significan$c i a=.000$; se acepta que la calidad de la información promueve un impacto positivo en los usuarios con información más relevante y rápida, lo que ayuda a evaluar alternativas para la toma de mejores decisiones.

$\mathrm{H}_{4}$ con un valor de $\mathrm{R}=.760, \mathrm{R}^{2}=.580$ y significancia $=.000$; a partir de ello, se acepta que la calidad de la información produce que los usuarios tengan un impacto positivo en el uso óptimo de los reportes, ayudando a mejorar el desempeño y la efectividad proveyendo información útil.

Por último, se rechaza $\mathrm{H}_{1}$, debido a que sus valores de $R=.415, R^{2}=.178$ y significancia $=.081$, solo cumplen con dos de los criterios aceptables $\left(R\right.$ y $\left.R^{2}\right)$, donde se propone que la administración de la información permite que los usuarios de la información contable, tomen más y mejores decisiones, lo que es desechado porque, tener una buena administración de la información no necesariamente permite tomar más decisiones de las se deban o se ignora cómo crearlas a partir del uso de un SIC.

\section{Conclusiones}

El objetivo de la presente investigación fue determinar el grado de influencia que tienen la administración y la calidad de la información en los sistemas de información contable que usan las pequeñas y medianas empresas, lo que les ayuda para la toma de decisiones y hacer un mejor uso/utilidad por los usuarios.

Por lo que se pudo obtener, la administración de la información juega un papel primordial, y en los últimos años las empresas han visto que el contar con buena información es más importante de lo considerado en el pasado, por lo que se han dado a la tarea de estudiar técnicas para el adecuado uso y manejo, y con la ayuda de las tecnologías, los usuarios hacen un mayor y mejor uso de la información generada en reportes, además de incrementar la efectividad por parte de los usuarios en el trabajo.

Asimismo, la calidad de la información proporciona a los usuarios datos útiles, rápidos y confiables, permitiéndoles evaluar de forma segura más alternativas disponibles para su uso óptimo. Todo esto les ayuda a hacer más eficiente la toma de decisiones con información útil y rápida, mejorando el uso y su efectividad. 
Por otro lado, se desecha el supuesto de que la administración de la información permite que los usuarios de la información contable, cuenten con más información relevante que les permita evaluar alternativas y tomar más, mejores y rápidas decisiones; es decir, no se está llevando a cabo una adecuada gestión de la información en beneficio de tomar decisiones que impacten el desarrollo armonioso de la organización.

Por último, se observa que en los últimos años, las PYMES le han dado cierta importancia al manejo de la información, pero como se comprueba, aún no han aprendido a aprovecharla para alcanzar una administración estratégica. Podría decirse que sólo están respondiendo a las necesidades del mercado, pero su uso y aplicación no es de una manera sistemática. Por lo tanto, y como líneas futuras de investigación, se cuenta con dos vertientes: i) es necesario que las PYMES repiensen sus procesos de negocio en cuanto al manejo de la información, y ii) analizar las causas del porqué la administración de la información no está ayudando a la toma de decisiones organizacionales. Lo anterior, debido a que no ha sido utilizada para la toma de decisiones, y más aun, si en el mundo globalizado en el cual se vive, la nueva tendencia es la administración del conocimiento, con estos resultados que son el reflejo de la zona bajo estudio, no se puede avanzar, si no está saldada eficientemente la deuda pendiente de la administración de la información.

\section{Referencias}

Anderson, J. \& Narasimhan, R. (1979). Assessing project implementation risk: a meth:odological approach. Management Science, 25 (6) (pp. 512-521).

Auer, T. \& Rouhonen, M. (1997). Analysing the quality of IS use and management in the organizational context: experiences from two cases. Information Resources Management Journal, 10 (3) (pp. 18-27).

Ballou, D., Wang, R., Pazer, H. \& Tayi, G.K. (1998). Modeling information manufacturing systems to determine information product quality. Management Science, 44 (4) (pp. 462-484).

Best, D. (2010). The future of information management. Records Management Journal, 20 (1) (pp. 61-71).

Cheney, P.H. \& Dickson, G.W. (1992). Organizational characteristics and information systems: an exploratory investigation. Academy of Management Journal, 25 (1) (pp. 170-184).

Chin, W.W. (1998). Issues and opinion on structural equation modeling. MIS Quarterly, 22 (1) (pp. vii-xvi).

Davenport, T.H. (1997). Information ecology. Mastering the information and knowledge environment. New York, USA: Oxford University Press.

Davis, F.D. (1986). A technology acceptance model of empirical testing new end-user information systems: theory and result. Tesis Doctoral. Sloan School of Management (MIT), Cambridge, MA, USA. [Doctoral Dissertation not printed].

Davis, F.D. (1989). Perceived usefulness, perceived ease of use, and user acceptance of information technology. MIS Quarterly, 13 (3) (pp. 319-340).

DeLone, W.H. \& McLean, E.R. (2002). Information systems success revisited. Proceedings: 35th Hawaii International
Conference on System Sciences. Computer Society Press. Hawaii, USA, 7-10 January.

DeLone, W.H. \& McLean, E.R. (2003). The DeLone and McLean model of information systems success: a ten-year update. Journal of Management Information Systems, 19 (4) (pp. 9-30).

English, L.P. (2001). Information quality management: the next frontier. Milwaukee, USA: American Society for Quality's 55th Annual Quality Conference Proceedings.

Franz, C.R. \& Robey, D. (1986). Organizational context, user involvement, and the usefulness of information systems. Decision Sciences, 17 (3) (pp. 329-356).

Gelderman, M. (1998). The relation between user satisfaction, usage of information systems and performance. Information \& Management, 34 (1) (pp. 11-18).

Goff, J. (2003). Drowning in data. CFO Magazine, 19 (11) (pp. 97-102).

Huber, G.P. \& McDaniel, R.R. (1989). The decision-making paradigm of organizational design. Management Science, 32 (5) (pp. 572-589).

Igbaria, M., Guimaraes, T. \& Davis, G.B. (1995). Testing the determinants of microcomputer usage via a structural equation model. Journal of Management Information Systems, 11 (4) (pp. 87-114).

Ives, B., Olson, M.H. \& Baroudi, J.J. (1983). The Measurement of User Information Satisfaction. Communications of the ACM, 26 (10) (pp. 785-793).

Jeske, D. \& Zhang, X. (2005). Some successful approaches to software reliability modeling in industry. The Journal of Systems and Software, 74 (1) (pp. 85-99).

Juran, J.M. \& Godfrey, A.B. (1999). Juran's quality handbook $5^{\text {th }}$ ed. New York, USA: McGraw Hill.

Koontz, H. y Weihrich, H. (2004). Administración. Una perspectiva global. México: Ed. McGraw Hill.

Krishnan, R., Peters, J., Padman, R. \& Kaplan, D. (2005). On data reliability assessment in accounting information system. Information Systems Research, 16 (3) (pp. 307326).

Leidner, D.E., Carlsson, S., Elam, J.J. \& Corrales, M. (1999). Mexican and Swedish Managers' Perceptions of the Impact of EIS on Organizational Intelligence, Decision Making, and Structure. Decision Science, 30 (3) (pp. 633-658).

Lillrank, P. (2003). The quality of information. International Journal of Quality \& Reliability Management, 20 (6) (pp. 691-703).

Marchand, D., Kettinger, W. \& Rollins, J. (2002). information orientation: the best link to business performance. New York, USA: Oxford University Press.

Markus, M.L. \& Keil, M. (1994). If we build it, they will come: designing information systems that people want to use. Sloan Management Review, 35 (4) (pp. 11-25).

Mathieson, K. \& Chin, W.W. (2001). Extending the technology acceptance model: the influence of perceives user resources. The DATA BASE for Advances in Information Systems, 32 (3) (pp. 86-112).

Medina, M. \& Chaparro, J. (2007). The impact of the human element in the information systems quality for decision making and user satisfaction. Journal of Computer Information Systems, 48 (2) (pp. 44-52).

Miller, J. \& Doyle, B.A. (1987). Measuring the effectiveness of computer-based information systems in the financial services sector. MIS Quarterly, 11 (1) (pp. 107-124). 
Molla, A. \& Licker, P.S. (2001). E-Commerce systems success: an attempt to extend and respecify the DeLone and McLean Model of IS Success. Journal of Electronic Commerce Research, 2 (4) (pp. 131-141).

Nunnally, J. (1978). Psychometric theory. New York, USA: McGraw Hill Editorial.

Organisation for Economic Co-operation and Development (OECD). (2004). OECD Digital Economy Papers, № 79, doi: $10.1787 / 232751878060$.

Oppenheim, Ch., Stenson, J. \& Wilson, R.M.S. (2004). Studies on information as an Asset III: views of information professionals. Journal of Information Science, 30 (2) (pp. 181-190).

O’Reilly, C.A. III. (1982). Variations in decision makers' use of information sources: the impact of quality and accessibility of information. Academy of Management Journal, 25 (4) (pp. 756-771).

Rai, A., Lang, S.S. \& Welker, R. (2002). Assessing the validity of is success models: an empirical test and theoretical analysis. Information Systems Research, 13 (1) (pp. 50-69).

Robbins, S., Coulter, M. (2010). Administración. $10^{\mathrm{a}}$ ed. México D.F.: Prentice Hall.

Sääksjärvi, M. \& Talvinen, J.M. (1993). Integration and effectiveness of marketing information systems. European Journal of Marketing, 27 (1) (pp. 64-79).

Serafeimidis, V. (2002). A review of research issues in evaluation of information systems. En: Van Grembergen, W.
(Ed.). Information systems evaluation management. London, United Kingdom: IRM Press (pp.167-194).

Sherman, B.A., Garrity, E., Kim, Y. \& Sanders, G.L. (2004). A model of information systems success. working paper. Buffalo, New York, USA: Canisius College.

Shin, N. (2001). The impact of information technology on financial performance: the importance of strategic choice. European Journal of Information Systems, 10 (4) (pp. 227-236).

Shin, B. (2003). an exploratory investigation of system success factors in data warehousing. Journal of the Association for Information Systems, 4 (6) (pp. 141-170).

Srinivasan, A. (1985). Alternative measures of system effectiveness: associations and implications. MIS Quarterly, 9 (3) (pp. 243-253).

Straub, D., Limayem, M. \& Karahanna-Evaristo, E. (1995). Measuring system usage: implications for is theory testing. Management Science, 41 (8) (pp. 1328-1342).

Teng, J.T. \& Calhoun, K.J. (1996). Organizational computing as a facilitator for operational and managerial decision making: an exploratory study of managers' perceptions. Decision Sciences, 27 (4) (pp. 673-710).

Teo, T. \& Wong, P.K. (1998). An empirical study of the performance impact for computerization in the retail industry. Omega, 26 (5) (pp. 611-621).

Tzu-Chuan, C., Dyson, R.G. \& Powell, P.L. (1998). An empirical study of the impact of information technology intensity in strategic investment decisions. Technology Analysis \& Strategic Management, 10 (3) (pp. 325-339).

Cuadernos de Administración / Facultad de Ciencias de la Administración / Universidad del Valle Periodicidad: semestral / ISSN impreso Nº120-4645-ISSN electrónico N²256-5078 / Nombre abreviado: cuad.adm. Edición Vol. $29 \mathrm{~N}^{\circ} 49$ (enero - junio de 2013) Administración y calidad de la información de los sistemas de información contable de las PYMES / José M. Medina-Quintero y Perla E. Aguilar-Gámez 